screened the allelic frequencies of $(G T)_{n}$ repeats in the HO-1 gene promoter in these obese children. NAFLD was determined through liver ultrasonography. Because the distribution of numbers of $(\mathrm{GT})_{\mathrm{n}}$ repeats was bimodal, we divided the alleles into 2 subclasses: class $\mathrm{S}$ included shorter ${ }_{\mathrm{n}}$ repeats in HO-1 gene promoter on paediatric NAFLD.

Results Of 101 obese subjects, 27 (26.7\%) had NAFLD. The alanine aminotransferase (ALT) level was higher in patients carrying $\mathrm{L}$ alleles $(\mathrm{L} / \mathrm{L}$ and $\mathrm{L} / \mathrm{S})$ than patients with $\mathrm{S}$ alleles $(\mathrm{S} / \mathrm{S})$ $[46.2 \pm 49.3 \mathrm{IU} / \mathrm{L}$ versus $30.2 \pm 20.1 \mathrm{IU} / \mathrm{L}]$. The significant risk factors for paediatric NAFLD were patients carrying L alleles (L/ $\mathrm{L}$ and $\mathrm{L} / \mathrm{S})(11.613 ; 95 \% \mathrm{CI}, 1.2$ to $112.43 ; \mathrm{p}=0.034)$, and HOMAR-IR (1.37; 95\% CI, 1.05 to $1.79 ; \mathrm{p}=0.019)$.

Conclusions In this hospital-based study, the obese children with larger GT repeats and insulin resistance were susceptibility to have NAFLD.

\section{PS-075 GENETIC COUNSELING OF WILSON DISEASE IN EGYPTIAN CHILDREN: EVALUATION OF ATP7B GENE MUTATION AS A USEFUL TOOL}

E Salama ${ }^{1}$, E Salama ${ }^{2}$, F Tarek ${ }^{2}$, H Hala ${ }^{3},{ }^{3}$ A Ayman, ${ }^{3}$ A Manar. ${ }^{1}$ Pediatric Department, Menoufia University, Cairo, Egypt; ${ }^{2}$ Pediatric Department, Menoufia University, Cairo, Egypt; ${ }^{3}$ Biochemistry Department, Menoufia University, Cairo, Egypt

\subsection{6/archdischild-2014-307384.372}

Backgrounds and aims Wilson disease (WD) is an autosomal recessive disorder of copper transport, $\mathrm{ATP} 7 \mathrm{~B}$, the gene mutated in WD, consists of 21 exons and encodes a 1,465 amino acid protein representing a copper transporting P-type adenosine triphosphatase (ATPase). Our aim was to evaluate His1069Gln mutation in exon 14 of ATP7B gene as a useful tool in genetic counseling.

Methods This study was conducted on 20 index cases with WD (group I) and their siblings (group II, $\mathrm{n}=27$ ), 47 apparently healthy children as control group (group III). Diagnosis of WD was made if the patient had hepatic and/ or neurologic disease in addition to at least two of the following six criteria: Positive family history of WD, Low ceruloplasmin level $<20 \mathrm{mg} / \mathrm{dl}$., Presence of kyser- Fleisher ring, Liver biopsy suggestive of WD, Elevated 24 hour urinary copper excretion and finally Coomb's negative hemolytic anemia. Molecular testing for ATP7B (His1069Gln) done by PCR based on restricted fragment length polymorphism assay.

Results It was found that the frequency of the His1069Gln gene mutation was 25\% among index cases group, $14.8 \%$ among siblings of the index group. As regard genotype- phenotype association there was no statistically significant difference between different types of disease phenotype regarding the mean age of onset, gender and presence of ATP7B gene mutation.

Conclusions The His1069Gln of ATP7B gene mutation could be used as a detection tool for WD in high risk population.

\section{PS-076 NOVEL MUTATIONS UNDERLYING RARE FAMILIAL ENTEROPATHIES}

A Almehaidib. Pediatrics, King Faisal Specialist Hospital and Research Center, Riyadh, Saudi Arabia

\subsection{6/archdischild-2014-307384.373}

Background Microvillous Inclusion Disease (MVID) and Congenital Tufting Enteropathy (CTE) are congenital disorders of the intestinal epithelial cells that cause an intractable diarrhoea since birth. MVID and CTE are very rare disorders and inherited as autosomal recessive traits. Recently, mutations of MYO5B and EpCAM were identified as the underlying lesion resulting in MVID and CTE, respectively.

Method Four Saudi families were investigated, three with children affected by MVID and one with a child affected by CTE. Five patients and available unaffected individuals were subjected to genome-wide homozygosity scans using the Affymetrix 250K SNP array. Analysis with the copy number tool CNAG identified shared homozygous regions unique to the affected subjects.

Results Of the three families with MVID, homozygosity was observed in two families at a locus on chromosome 18 which included MYO5B. Sequencing of MYO5B in individuals from these families identified two novel nonsense mutations in exons 24 and 36 (Q1047X and E1589X). In the other family homozygosity was absent at the MYO5B locus. However, a locus on chromosome 2 which included EDCAM was found to be homozygous in this family. Sequencing of EpCAM identified a $1 \mathrm{bp}$ insertion (c.499insC) in exon 5 resulting in premature truncation of the mature protein. This was consistent with this family being classified as having CTE rather than MVID. The fourth family studied was referred with a diagnosis of CTE and was found to have the same $1 \mathrm{bp}$ insertion consistent with a common founder.

Conclusion We have identified novel nonsense mutations in $M Y O 5 B$ and EpCAM associated with congenital enteropathies in Saudi families. Our findings expand the limited spectrum of $M Y O 5 B$ and EpCAM mutations associated with gastrointestinal genetic disorders and provide an opportunity to investigate phenotype/ genotype correlations.

\section{PS-077 THE IMPLICATION OF IL-6 572 C/G GENE POLYMORPHISMS IN CHILDREN'S MALNUTRITION}

${ }^{1} \mathrm{O}$ Marginean, ${ }^{1} \mathrm{M}$ Chincesan, ${ }^{2} \mathrm{M}$ Marginean, ${ }^{3} \mathrm{C}$ Marginean. ${ }^{1}$ Pediatrics, University of Medicine and Pharmacy, Tirgu Mures, Romania; ${ }^{2}$ Pediatrics, University of Medicine and Pharmacy, Targu Mures, Romania; ${ }^{3}$ Obstetrics, University of Medicine and Pharmacy, Targu Mures, Romania

\subsection{6/archdischild-2014-307384.374}

Malnutrition is a disorder caused by reducing the energetical and/or protein input, with adverse effects on tissue and body form and function and different clinical consequences.

Aim

The aim of this study was to establish the correlations between the polymorphisms of the IL-6 572 gene in malnourished children.

Methods We assessed 283 hospitalised children divided into two groups: group I (control) included 110 patients with normal nutritional status, and group II consisted of 173 malnourished patients.

The two groups underwent IL-6 572 C/G polymorphism testing, measurement of anthropometric parameters (mid-upper arm circumference, MUAC and tricipital skinfold thickness, TST), and paraclinical evaluation (protein, albumin).

Results We observed that the differences between the anthropometric parameters (MUAC and TST) corresponding to the three types of genotypes (CC, CG, GG) were statistically significant lower in malnourished children with all the three genotype of IL-6 572 ( $\mathrm{p}=0.0001)$. Same correlations were noticed with low serum albumin levels. 